https://www.journal-imab-bg.org

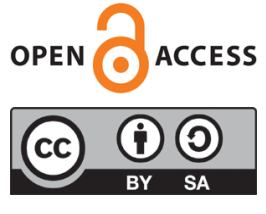

Original article

\title{
BEHAVIORAL AND NUTRITIONAL ASSESSMENT OF TEENAGERS FROM VARNA.
}

\author{
Dimitar B. Marinov ${ }^{1}$, Darina N. Hristova ${ }^{2}$ \\ 1) Department of Hygiene and Epidemiology, Faculty of Public Health, Medi- \\ cal University of Varna \\ 2) Department of Preclinical and Clinical Sciences, Faculty of Pharmacy, Medi- \\ cal University of Varna, Bulgaria.
}

\begin{abstract}
:
Adolescence is a key stage of life through which the patterns for physical activity, nutrition, and other health habits form.

Aim: Evaluating the eating behavior of 401 highschool students from varna studying in 12 th grade $(11.2 \%$ of the whole group, used as a representative sample).

Methods: FFQ (food frequency questionnaire) on 37 food groups, questionnaire for eating attitude, IPAQ (validated questionnaire for the physical activity in the last 7 days), anthropometrical measurements (height, weight, $\mathrm{BMI})$.

Results: Roughly $70 \%$ of students in 12 th grade who are underweight, overweight (OW), or obese ate regular breakfast, compared to $84 \%$ of those with normal weight. This was the most commonly skipped meal of the day. We found a statistically significant correlation between BMI in boys and eating breakfast regularly. $\left(\mathrm{r}=-0.473^{*} *, \mathrm{p}<0.001\right)$. Skipping breakfast is quite common $(49,7 \%)$ among OW girls. Its correlation with BMI is also significant ( $\mathrm{r}=-0.492 * *$, $\mathrm{p}<.001$ ). White bread and other bakery products are consumed very often by both genders. Consumption of fruits and vegetables, milk $(51,3 \%)$, and fish $(68 \%)$ is low amongst adolescent girls. In contrast, male students have at least 2 portions of milk or milk products daily $(94,5 \%)$. This finding correlates positively with their BMI $(\mathrm{r}=0.401 * *, \mathrm{p}<0.001)$. There is a normal BMI in $60.7 \%$ of female and $67.2 \%$ of male students $\left(18,5-24,9 \mathrm{e} g / \mathrm{m}^{2}\right) .23,5 \%$ of female students in 12 th grade are underweight, especially those with physical activity that is higher than average $(38,9 \%)$.

Conclusion: Large percentage of the female students in 12 th grade wish to lose bodyweight, including those in the underweight group. Low self-esteem and restrictive dieting can increase the risk of eating disorders in young girls.
\end{abstract}

Keywords: adolescents, eating attitude, eating disorders, food frequency,

\section{INTRODUCTION:}

Adolescence is a key stage of life, through which significant biological, physiological, social, and cognitive changes occur. Limiting factors for the normal manifestation of these processes are adequate nutrition and optimal physical activity (PA). Adolescence is a critical period in terms of establishing a certain model of behavior including the formation of unhealthy habits [1]. Routines, in regards to nutrition and PA in this age, are very persistent in time and can be a precursor of the health for the rest of the life. In high-school age is manifested an autonomy of decision making in regards to lifestyle (food, sport, alcohol, tobacco, drugs, etc.). Self-esteem in this age is strongly influenced by body shape and weight, which leads to a desire to achieve "ideal" weight and body. Among teenagers in Bulgaria and around the world, there are widely spread unhealthy dietary practices $[2,3]$. The unfavorable model of nutrition in the high-school age is caused by multiple factors, including the lack of a healthy lifestyle in the family and school environment, the "deficit" in the health culture and in particular in the culture of nutrition in society, and the lack of adequate health education in schools $[4,5,6]$.

This study aims to reveal the characteristics of eating habits in high school students. The subject of the survey is a group of 401 students studying in the Varna region. The frame sample is 3553 students from 12th grade in the Varna district according to the RIE. A random sample of $11.2 \%$ of the target group was enrolled to achieve the representativeness of the study.

A set of methods for assessing dietary behavior was used:

- Tests and procedures related to nutritional status assessment: anthropometric indicators and indexes - growth, weight, body mass index (BMI), bioelectric impedance analysis (BIA)

- A questionnaire study of eating attitude (diet, nutritional preferences, frequency of food consumption of 37 categories of foods, etc.) 
- Physical activity has been evaluated for the last week via a validated international questionnaire IPAQ [7].

\section{RESULTS:}

\section{Dietary regime.}

Nutrition in youngsters is healthy when it includes at least three basic dietary intakes for the day and the possibility of one or two snacks. Nearly $84 \%$ of adolescents with normal body weight, evaluated on a BMI basis, have regular breakfast each morning, in contrast to $70 \%$ of their classmates who are underweight, overweight $(\mathrm{OW})$, or obese. There is a significant negative relationship between morning breakfast and BMI in young men $(\mathrm{r}=-0.473 * *, \mathrm{p}$ $<0.001)$. Young people with optimal bodyweight are twice as likely to eat in the afternoon (38.3\%), as opposed to their overweight peers (20.7\%). Dietary intake for students that are overweight or obese is shifted to the second half of the day, with a preference for lunch, afternoon snacks, and mandatory dinners, whereas for optimal and normal body weight, diet usually includes breakfast and a second breakfast. The feeding regime in females shows significant differences depending on their degree of nourishment. Girls with optimal weight more commonly eat in the morning $-71.2 \%$ vs. $65.1 \%$ of the underweight group $(\mathrm{p}<0.05)$. Much of the underweight students have an optimal diet, and the reason for their inadequate nourishment can be found in their nutritional choices, the size of the portion, and the level of PA that significantly affects energy expenditure. Only half of the overweight girls $(50.3 \%)$ include morning breakfast in their daily diet and a small portion of them have a supportive meal before noon $(10.3 \%)$. The majority of girls often eat twice a day - at lunch and dinner, and usually miss breakfast, brunch, or afternoon meal. Female students that are overweight or obese often say they deliberately do not eat until lunch to "better control their appetite during the day" and reduce bodyweight. There is a significant negative correlation between the presence of morning breakfast and BMI in young women ( $\mathrm{r}$ $=-0.492 * *, \mathrm{p}<.001)$.

Fig. 1. Dietary regime - girls

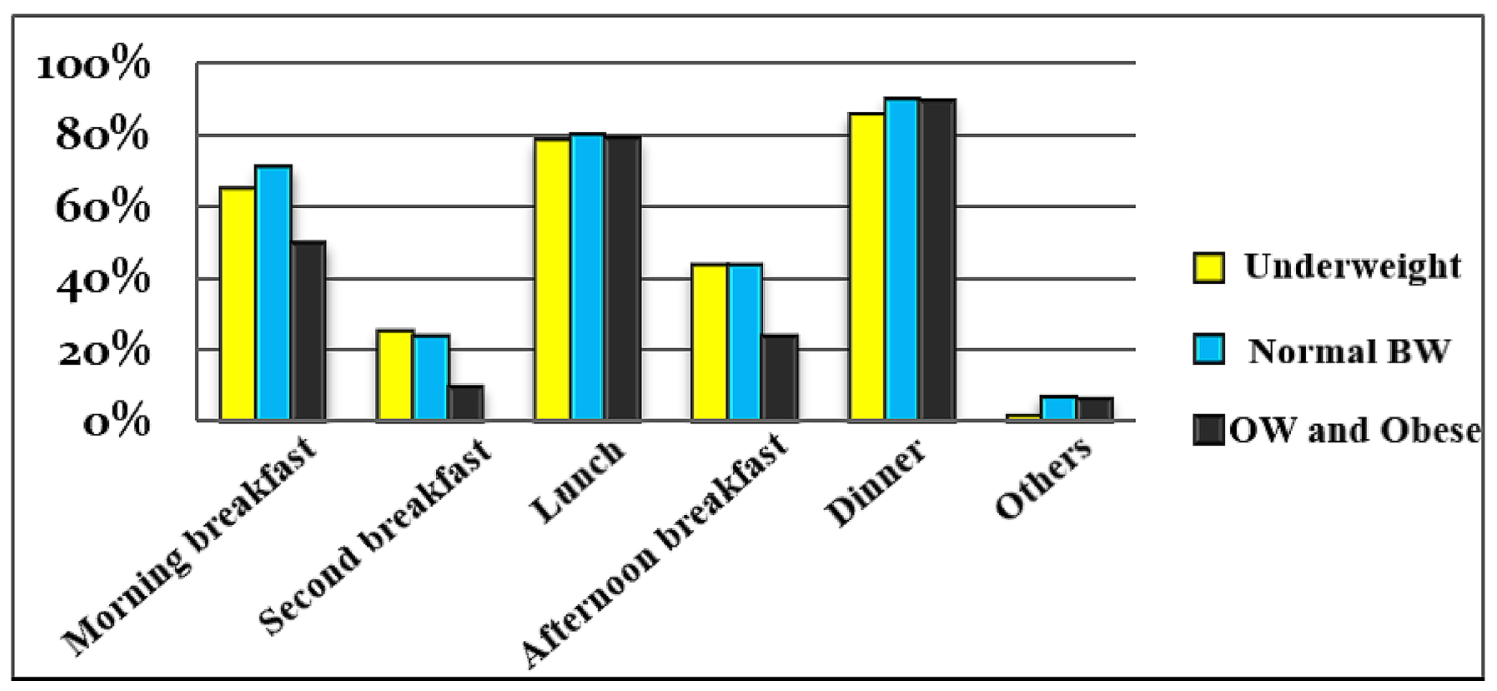

A basic requirement for breakfast is to import 20$35 \%$ of daily energy needs. A breakfast that is optimal for health and bodyweight includes low glycemic index foods, sources of protein, and essential fat. An unbalanced breakfast adversely affects satiety, the control of appetite, and ultimately the bodyweight. Conditions for a caloric dense and balanced breakfast are not met when only fruits or vegetables are consumed or when breakfast is replaced with bakery products or only pastry. The morning breakfast of underweight girls is usually highly energy-dense (pasta, sandwich). Adding fresh fruit or vegetable to the sandwich or dessert will greatly improve the biological value of the morning breakfast, but this is only a habit of $16.3 \%$ of underweight girls. Only $18.9 \%$ of girls with normal body weight have a well-balanced breakfast, which includes fresh fruits or vegetables. Young overweight and obese women are the least likely to eat pastry and confectionary at breakfast (31\% and $6.9 \%$ respectively) and most often eat fruits or vegetables (37\%). The absence of pasta and sweet foods in the menu of those girls probably reflects the underreporting characteristic of people with obesity (declaring lower dietary consumption than the actual high-calorie foods). On the other hand, these data could also be explained by deliberate restraint to lower bodyweight. 
Fig. 2. Breakfast choices - girls

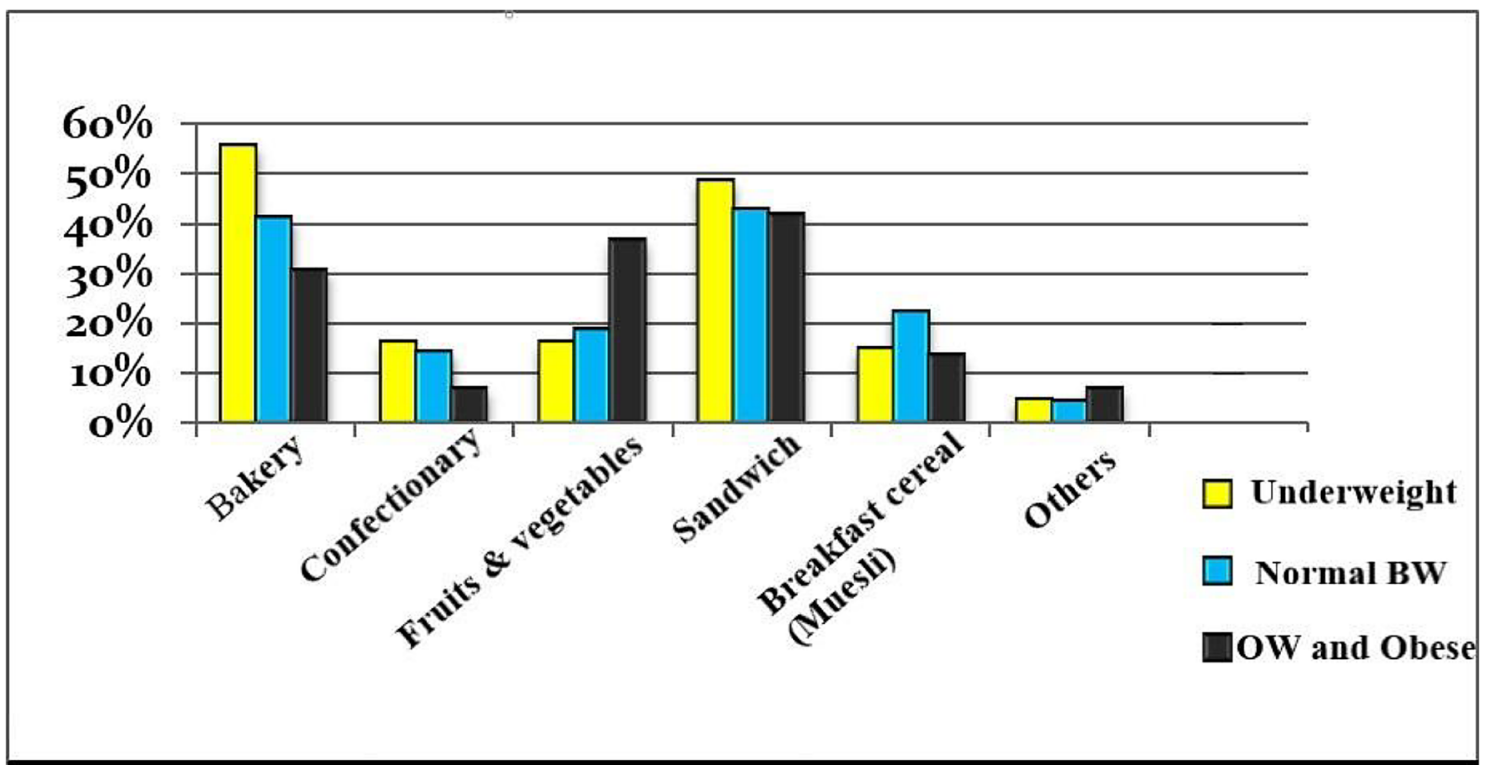

\section{Nutritional preferences}

A diverse diet, along with adequate nutrition, is the heart of healthy eating. Regular consumption of foods of high biological value reduces the risk of eating deficiencies. The calorie of the two main types of bread (white and whole grain) is very similar, but there are serious differences in their biological value (fiber and B vitamins). Over $80 \%$ of the subjects in our study prefer white bread. Over $41 \%$ of students consume pastries daily, which are a source of trans fats, excess of salt and calories. It is recommended that each daily intake should include a portion of fresh fruit and vegetables to provide for the needs of vitamin $\mathrm{C}$ and folate. Only $15.2 \%$ of youngsters consume at least two servings of vegetables a day as recommended, and $23.9 \%$ - include vegetables in their menu once a day. Low consumption amongst girls puts them in a situation of inadequate folate intake, which is essential for ovarian function. Another unfavorable trend in adolescents eating patterns is the low consumption of fish. Only around $1 / 3$ of respondents have an adequate fish intake, consuming it at least 1-2 times a week. The rest of the participants which includes $63.1 \%$ for boys and $68 \%$ for girls have low fish consumption and are likely to have a shortage of essential fatty acids (Ù-3) and during the winter season - vitamin
D. Consumption of enough milk helps children and adolescents to reach the genetically set peak bone mass and reduces the risk of osteoporosis. The recommendation is to consume at least 2 portions of milk and dairy products daily (2 servings equal $400 \mathrm{ml}$ of milk or yogurt or $100 \mathrm{~g}$ of cheese or yellow cheese). This recommendation was met in $94.5 \%$ of young men and only in $48.7 \%$ of girls. More than half of the students have a low intake of riboflavin and calcium. The lowest consumption is in the underweight group (Figure 3). A higher frequency of dairy consumption ( $>1$ serving daily) correlates positively with BMI in adolescents $(\mathrm{r}=0.401 * *, \mathrm{p}<0.001)$. The drink most commonly consumed by students, aside from water, is coffee. Daily coffee are drinking $48.6 \%$ of girls and $42.9 \%$ of boys. There is high consumption of carbonated beverages $(26 \%$ of young people consume them daily) and energy drinks (13\% of young people consume them daily). Every day, $34.4 \%$ of girls drink juices. Compared to the low fruit consumption in the group of girls, the preference for juices is linked on one hand with lower dietary intake of folate and fiber and, on the other hand, with the addition of extra calories in the form of simple sugars and poor control of appetite due to the high glycemic index of these beverages. 
Fig. 3. Consumption of milk and dairy foods - males

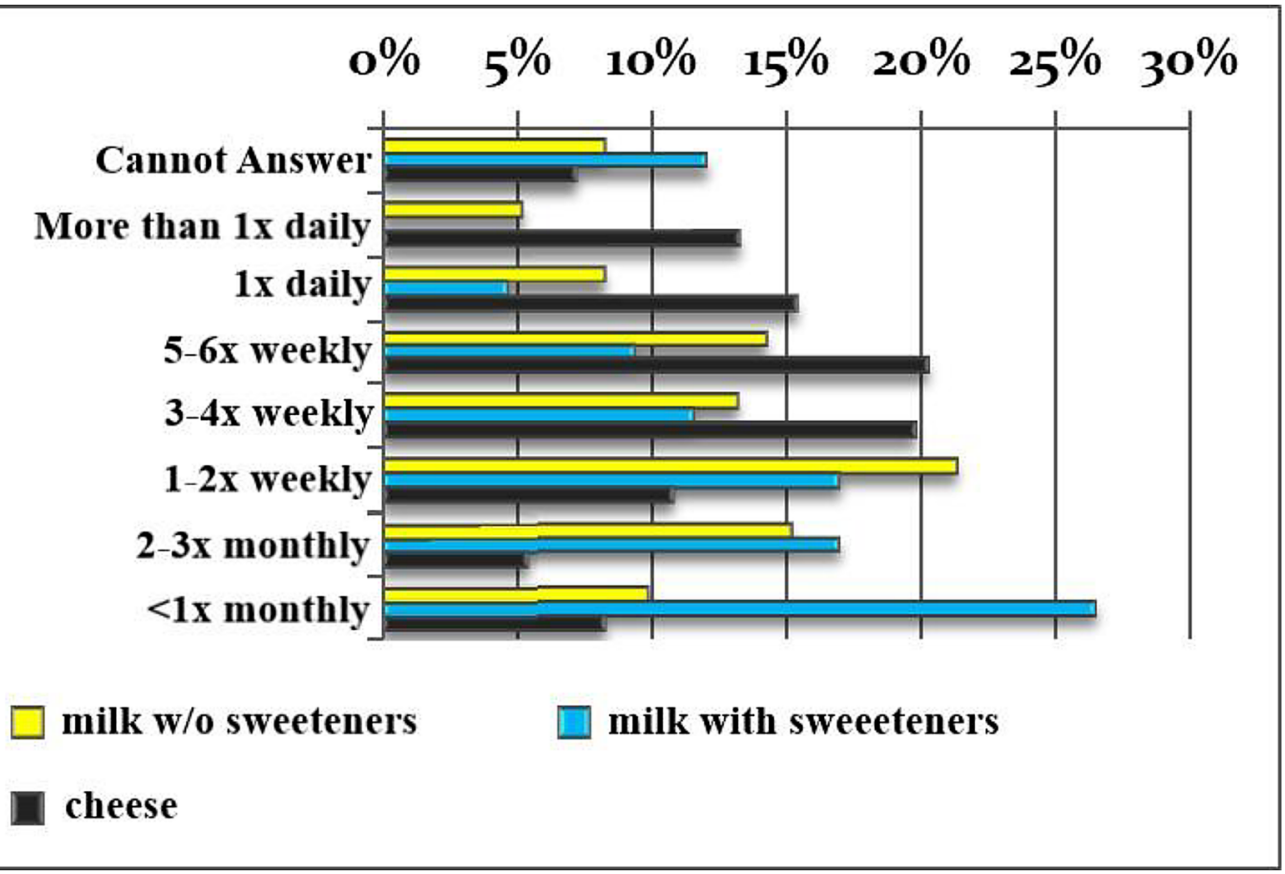

BMI analysis and PA levels:

The categories of being normal, underweight, or overweight are based on BMI. About $2 / 3$ of the studied subjects (67.2\% of the boys and $60.7 \%$ of the girls) have a normal BMI value of $18.5-24.9 \mathrm{~kg} / \mathrm{m} 2$. The incidence of underweight and overweight and obesity in both genders is similar. Young women with underweight are nearly a quarter of the whole representative sample $(23.5 \%)$. The highest underweight prevalence is in girls with high PA - 38.9\%. This result is troublesome because low bodyweight means less variety and more restrictions in their diet. In the course of direct contact with the girls, the study found problems related to their mental functioning. Regardless of the bodyweight changes and the specificities of their body composition, a significant proportion of the young women shared dissatisfaction with their body weight and desire to lose weight. This problem is also present in the group of underweight girls who often state that their desired ideal weight is lower than their current weight by $2-4 \mathrm{~kg}$. The low self-esteem of adolescent girls is particularly troublesome because it provokes the occurrence of restrictive diets and eating disorders [8].

Fig. 4. Distribution of boys and girls by BMI

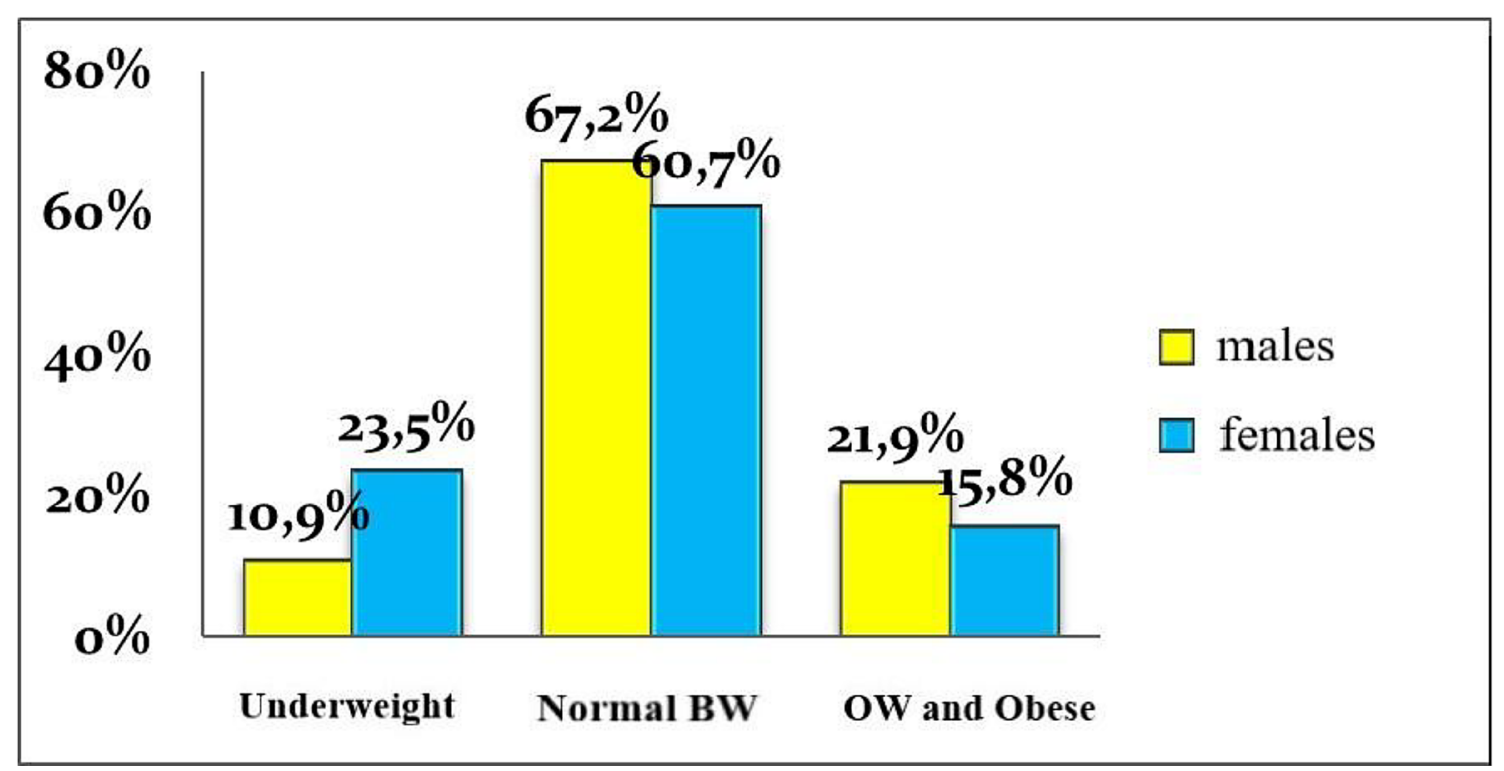




\section{CONCLUSION:}

1. The nutritional pattern of high-school students is characterized by some unfavorable features:

- insufficient consumption of foods with high biological value - whole grain bread, fresh fruit and vegetables, fish, milk, and dairy products (amongst girls)

- high consumption of carbonated beverages, juices (amongst girls), coffee and energy drinks (amongst boys)

- lack of breakfast in the group with obesity or overweight

2. There is a risk of inadequate dietary intake:

- amongst girls of vitamins C, B1, B2, B9, A, D, PUFA, calcium, fiber. The highest risk is in the underweight group

- amongst young men of vitamin D and PUFA
3. Analysis of BMI values showed:

- similar incidence of underweight and overweight or obese cases in both sexes.

- the high incidence of underweight $(23.5 \%)$ in females, especially in the high PA group $(38.9 \%)$

Limiting the health risks and reducing the incidence of nutritional deficiencies among younger generations can be achieved through intervention programs aimed at the media and the educational environment. An element of such a program would be the introduction of a mandatory training module on "Healthy Lifestyle" in schools. International experience has shown that the inclusion of training programs on behavioral risk factors in the professional training of staff in children's and educational establishments contributes to the creation of a health-promoting environment for adolescents.

\section{REFERENCES:}

1. Youth Risk Behavior Surveillance System (YRBSS). 2019 YRBS Results and Data Available Now. CDC. [Internet]

2. Chin SNM, Laverty AA, Filippidis FT. Trends and correlates of unhealthy dieting behaviours among adolescents in the United States, 19992013. BMC Public Health. 2018 Apr 17;18(1):439. [PubMed] [CrossRef]

3. Demissie Z, Lowry R, Eaton DK, Nihiser AJ. Trends in weight management goals and behaviors among 9th12th grade students: United States, 1999-2009. Matern Child Health J. 2015 Jan;19(1):74-83. [PubMed]
[Crossref]

4. Johnston DW, Lordan G. Weight perceptions, weight control and income: an analysis using British data. Econ Hum Biol. 2014 Jan;12:132-9. [PubMed] [Crossref]

5. Lowry R, Michael S, Demissie Z, Kann L, Galuska DA. Associations of Physical Activity and Sedentary Behaviors with Dietary Behaviors among US High School Students. J Obes. 2015; 2015:876524. [PubMed] [Crossref]

6. Miller GF, Sliwa S, Brener ND, Park S, Merlo CL. School District Policies and Adolescents' Soda Consump- tion. J Adolesc Health. $2016 \mathrm{Jul}$; 59(1):17-23. [PubMed] [Crossref]

7. Kurtze N, Rangul V, Hustvedt BE, Flanders WD. Reliability and validity of self-reported physical activity in the Nord-Trondelag Health Study: HUNT 1. Scand J Public Health. 2008 Jan;36(1):52-61. [PubMed] [Crossref]

8. Sonneville KR, Thurston IB, Milliren CE, Gooding HC, Richmond TK. Weight misperception among young adults with overweight/obesity associated with disordered eating behaviors. Int J Eat Disord. 2016 Oct; 49(10):937-946. [PubMed] [Crossref]

Please cite this article as: Marinov DB, Hristova DN. Behavioral and nutritional assessment of teenagers from Varna. $J$ of IMAB. 2021 Jan-Mar;27(1):35491-3553. DOI: https://doi.org/10.5272/jimab.2021271.3549

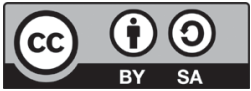

Address for correspondence

Dimitar Borisov Marinov

Department of Hygiene and Epidemiology, Medical University - Varna, 55, Marin Drinov Str., Varna, Bulgaria

E-mail: mitak_1992@abv.bg 